\title{
Heart failure with mildly reduced ejection fraction: emerging frontiers in clinical characteristics, prognosis, and treatment
}

\author{
Zhi Shang ${ }^{1}$, Xinyu Wang ${ }^{1}$, Wei Gao ${ }^{1, *}$ \\ ${ }^{1}$ Department of Cardiology and Institute of Vascular Medicine, Peking University Third Hospital, 100191 Beijing, China \\ *Correspondence: weigao@bjmu.edu.cn (Wei Gao) \\ Academic Editors: Brian Tomlinson and Takatoshi Kasai \\ Submitted: 6 September 2021 Revised: 8 October 2021 Accepted: 22 October 2021 Published: 18 January 2022
}

\begin{abstract}
Heart failure (HF) is a complex clinical syndrome resulting from the impairment of ventricular filling or ejection of blood or both, leading to considerable morbidity and mortality. Based on left ventricular ejection fraction (LVEF), the 2016 European Society of Cardiology (ESC) guideline firstly classified patients with LVEF in the range of $40 \%$ to $49 \%$ into heart failure with mid-range ejection fraction. Since then, more and more clinical studies targeting HF with mid-range ejection fraction emerged, indicating that they may benefit from similar therapies to those with $\mathrm{LVEF} \leq 40 \%$. So the latest ESC guideline of HF changed the term 'heart failure with mid-range ejection fraction' to 'heart failure with mildly reduced ejection fraction' (HFmrEF). Simultaneously, burgeoning evidence indicating the emergence of novel technologies (such as speckle tracking echocardiography, cardiac magnetic resonance quantitative imaging), and new biomarkers were conducive to evaluating HF from different perspectives. In this review, we summarized the research progress of HFmrEF in clinical characteristics, prognosis, and treatment, hoping to help cardiologists better evaluate and treat patients of HFmrEF.
\end{abstract}

Keywords: Heart failure with mildly reduced ejection fraction; Global longitudinal strain; T1 mapping; Extracellular volume fraction; Biomarkers; Prognosis; Treatment

\section{Introduction}

Heart failure (HF) is a clinical syndrome resulting from the dysfunction of ventricular systolic and/or diastolic function caused by various reasons, leading to an increasing disease burden worldwide. The mortality rate of HF remains high, with approximately $2-17 \%$ of $\mathrm{HF}$ patients died during hospitalization and $17-45 \%$ of $\mathrm{HF}$ patients died within 1 year after admission [1]. The prevention and treatment of HF are of vital significance. The 2016 European Society of Cardiology (ESC) guideline [2] divided HF into three categories based on left ventricular ejection fraction (LVEF). For the first time, the concept of HF with midrange ejection fraction was formally introduced, with the LVEF in the $40-50 \%$ range. Besides, patients with LVEF $\leq 40 \%$ and $\geq 50 \%$ were defined as HF with reduced ejection fraction (HFrEF) and HF with preserved ejection fraction (HFpEF), respectively. Since then, there have been more clinical studies targeting $\mathrm{HF}$ with mid-range ejection fraction. The latest 2021 ESC guideline [3] demonstrated patients with ejection fractions in the $40-50 \%$ range may benefit from similar therapies to those with LVEF $\leq 40 \%$, leading to the change of the term 'heart failure with mid-range ejection fraction' to 'heart failure with mildly reduced ejection fraction' (HFmrEF).

The mechanism of HFrEF was that the initial myocardial damage triggers persistent myocardial injury and myocardial remodeling, which eventually leads to a decrease in cardiac output [4]. HFpEF represented a complex syndrome, secondary to many different but interacting patho- physiological processes [5]. The phenotype and mechanism of HFmrEF were complex, with many characteristics between HFrEF and HFpEF [4,5]. Hence more comprehensive examination methods were needed for further analysis of HFmrEF. The emergence of more technologies is conducive to the analysis of patients with HF from different perspectives, such as speckle tracking echocardiography (STE), cardiac magnetic resonance (CMR) quantitative imaging, and new biomarkers, which can better distinguish the characteristics of different types of HF and contribute to diagnosis and prognosis. In this review, we summarized the research progress of HFmrEF in clinical characteristics, prognosis, and treatment, hoping to help cardiologists better evaluate and treat patients of HFmrEF.

\section{Epidemiology and clinical characteristics}

\subsection{Prevalence and etiology}

Since the term HFmrEF was first proposed in 2016, many clinical trials emerged, suggesting an HFmrEF prevalence of $12-25 \%$ among the overall HF population, which was less than HFrEF but comparable to HFpEF (Table 1, Ref. [6-11]). For etiology, Ischemic heart disease (IHD) was the leading cause of HF, while the proportion of IHD in HFrEF and HFmrEF was higher than HFpEF. A post hoc analysis of Trial of Intensified versus standard Medical therapy in Elderly patients with Congestive Heart Failure [7] demonstrated the proportion of IHD in HFrEF, HFm$\mathrm{rEF}$, and HFpEF was $58.2 \%, 56.5 \%$, and $31.3 \%$, respectively, indicating HFmrEF was similar to $\mathrm{HFrEF}$ in is- 
Table 1. Summarized results of landmark trials in patients with HFrEF, HFmrEF and HFpEF.

\begin{tabular}{|c|c|c|c|c|c|c|c|}
\hline Study & & Year & Patient number & Follow up (years) & HFrEF (\%) & HFmrEF (\%) & HFpEF (\%) \\
\hline \multirow{2}{*}{ Kapoor et al. [6] } & prevalence & \multirow{2}{*}{2016} & \multirow{2}{*}{99825} & \multirow{2}{*}{ inhospital } & 49 & 12.8 & 38.2 \\
\hline & mortality & & & & 3.06 & 2.62 & 3.02 \\
\hline \multirow{2}{*}{ Rickenbacher et al. [7] } & prevalence & \multirow{2}{*}{2017} & \multirow{2}{*}{622} & \multirow{2}{*}{2.2} & 65 & 17 & 18 \\
\hline & mortality & & & & 38 & 42 & 39 \\
\hline \multirow{2}{*}{ Chioncel et al. [8] } & prevalence & \multirow{2}{*}{2017} & \multirow{2}{*}{9134} & \multirow{2}{*}{1} & 59.8 & 24.2 & 16 \\
\hline & mortality & & & & 8.8 & 7.6 & 6.4 \\
\hline \multirow{2}{*}{ Koh et al. [9] } & prevalence & \multirow{2}{*}{2017} & \multirow{2}{*}{42061} & \multirow{2}{*}{3} & 56 & 21 & 23 \\
\hline & mortality & & & & 28.7 & 27.9 & 33 \\
\hline \multirow{2}{*}{ Löfman et al. [10] } & prevalence & \multirow{2}{*}{2017} & \multirow{2}{*}{40230} & \multirow{2}{*}{1} & 57 & 22 & 21 \\
\hline & mortality & & & & 14.8 & 14.7 & 18.5 \\
\hline \multirow{2}{*}{ Lund et al. [11] } & prevalence & \multirow{2}{*}{2018} & \multirow{2}{*}{7598} & \multirow{2}{*}{2.9} & 57 & 17 & 26 \\
\hline & mortality & & & & 30 & 15.8 & 16.6 \\
\hline
\end{tabular}

Abbreviations: HFrEF, heart failure with decreased ejection fraction; HFmrEF, heart failure with mildly reduced ejection fraction; HFpEF, heart failure with preserved ejection fraction.

chemic etiology. This was also confirmed by other studies [6,8,11-14]. Dilated cardiomyopathy (DCM) was another important cause of $\mathrm{HF}$, which accounted for about $1 / 3$ of the total number of patients, with HFmrEF and HFrEF significantly higher than HFpEF [7,8,14]. Differently, hypertensive heart disease and valvular heart disease were more common causes of HFpEF than the other two types [7,9]. Therefore, in terms of etiology, HFmrEF was more similar to HFrEF than HFpEF (Fig. 1, Ref. [7,8,11-13]).

\subsection{Comorbidities}

The prevalence of hypertension (HT) and atrial fibrillation (AF) in HFmrEF were higher than HFrEF [7-9,11]. In contrast, the prevalence of diabetes mellitus (DM) and chronic kidney disease (CKD) was comparable in HFmrEF and HFrEF. Compared with HFpEF patients, the prevalence of DM, anemia, AF, chronic obstructive pulmonary disease, and CKD was lower in HFmrEF [7-10]. Regarding prognosis, compared with $\mathrm{HFpEF}$, the correlation between $\mathrm{CKD}$ and mortality seemed to be stronger in HFrEF and HFmrEF, which may be due to the close relationship between CKD and the later stage of HF [10]. Similar to CKD, the effect of DM on mortality seemed to be greater in HFmrEF and HFrEF [15]. In contrast, AF had a similar impact on prognosis among all three types of HF [16].

\subsection{Prognosis}

Kapoor et al. [6] firstly analyzed the situation of 99,825 in-hospital patients, indicating that HFmrEF had the lowest in-hospital mortality of $2.62 \%$ while HFrEF accounted for $4.06 \%$ and HFpEF accounted for $3.02 \%$. With regard to long-term prognosis, emerging studies demonstrated that the mortality of HFmrEF was $7-42 \%$ with a median follow-up time ranged from 1 year to 3 years, which was similar to HFpEF and slightly lower than HFrEF [7-
11]. Therefore, we should attach more importance to the identification and management of HFmrEF. In terms of HF hospitalization rate, HFmrEF was similar to the HFpEF but significantly lower than HFrEF [8] (Table 1).

\section{Cardiac imaging}

\subsection{Echocardiography}

\subsubsection{Changes of left ventricular ejection fraction}

LVEF was the basis for the classification of HF. Prior studies have shown that when LVEF was less than $45 \%$, every $10 \%$ decrease in ejection fraction increased the risk of death by $39 \%$ [17]. However, LVEF in HF was not static but often changed over time. Only about $1 / 3$ of HFmrEF patients maintained a constant HF classification during longterm follow-up [12,18], while some of the remaining patients converted to HFrEF (25-37\%) and HFpEF (25-33\%) [19]. An observational study conducted by Lupón et al. [12] including 940 patients with baseline LVEF $<45 \%$ who performed echocardiography at one year follow-up showed that $24.8 \%$ of the patients had LVEF recovered to $\geq 45 \%$, which was called ejection fraction recovery HF (HFrecEF). However, this study used an empirical cut-off point of $45 \%$ to classify HF and did not further analyze patients of HFmrEF. Brann et al. [20] conducted a cohort study including $448 \mathrm{HFmrEF}$ patients who had a prior record of echocardiography, indicating LVEF improved from less than $40 \%$ in 157 patients (35.0\%), deteriorated from greater than $50 \%$ in 224 patients $(50.0 \%)$, and remained between $40 \%$ and $50 \%$ over time in 67 patients $(15.0 \%)$. But it was a retrospective study and deleted about half of HFmrEF patients who had not previously screened echocardiography, which may cause an offset. The SwedeHF study [18] including $3134 \mathrm{HFrEF}$ (63\%), $937 \mathrm{HFmrEF}$ (19\%), and $886 \mathrm{HFpEF}$ (18\%) patients at baseline indicated $16 \%$ HFrEF patients 


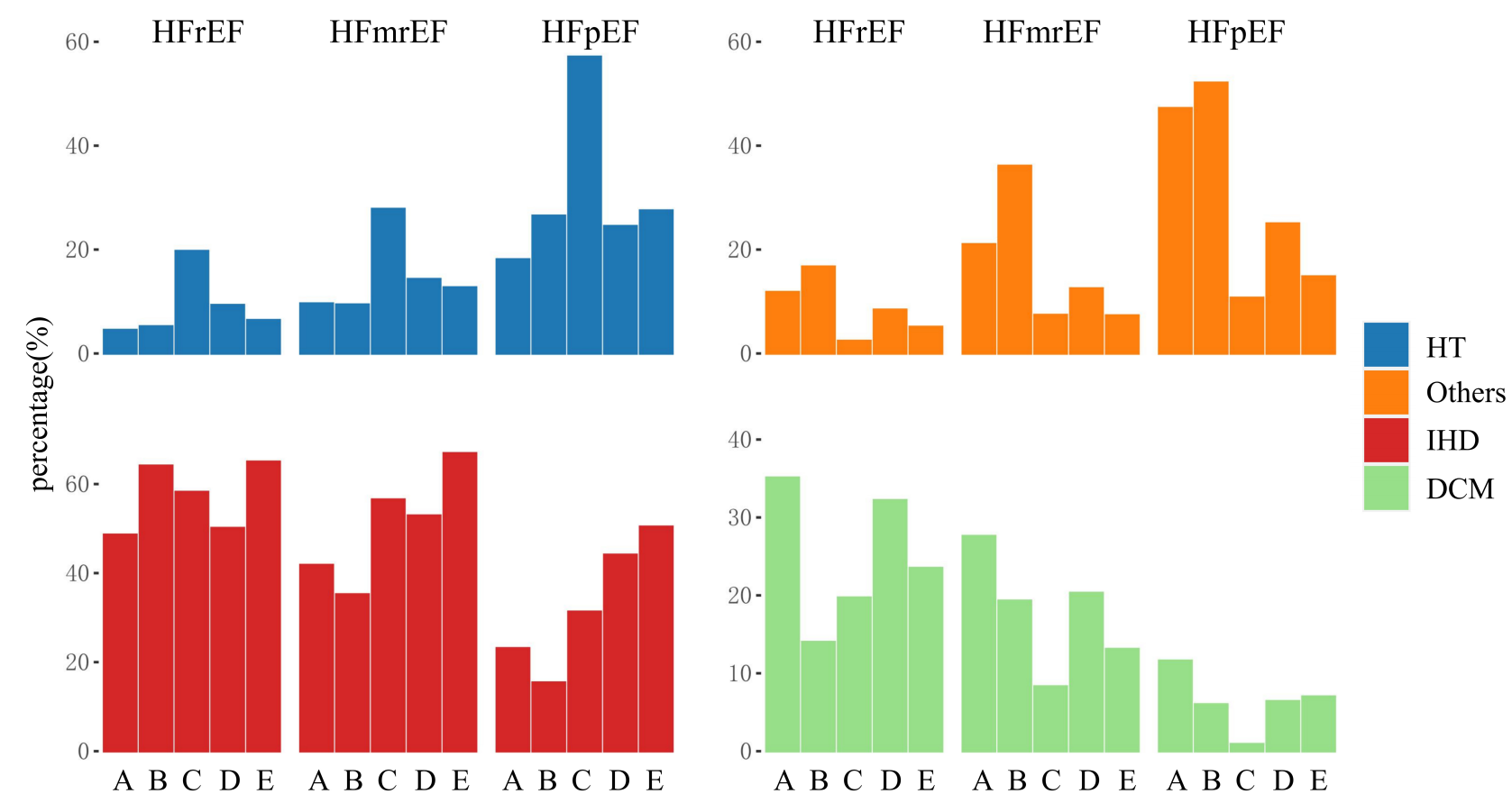

Fig. 1. Etiologies for HFrEF, HFmrEF and HFpEF. (A) 2017 Chioncel et al. [8]; (B) 2017 Lupón et al. [12]; (C) 2017 Rickenbacher et al. [7]; (D) 2018 Lund et al. [11]; (E) 2017 Tsuji et al. [13]. Abbreviations: HFrEF, heart failure with decreased ejection fraction; HFmrEF, heart failure with mildly reduced ejection fraction; HFpEF, heart failure with preserved ejection fraction; DCM, dilated cardiomyopathy; HT, hypertension; IHD, ischemic heart disease.

and $21 \% \mathrm{HFpEF}$ patients transferred to HFmrEF group during follow-up. And $38 \%$ of HFmrEF patients maintained their classification of HF. Because the number of patients with HFrEF was the largest, most of HFmrEF patients were converted from the increase of LVEF in HFrEF patients. A similar result was also found by Rastogi et al. [21]. This demonstrated that the majority of HFmrEF patients were caused by an improvement in HFrEF patients (Fig. 2, Ref. [18]).

3.1.2 The influence of left ventricular ejection fraction changes on prognosis

When LVEF changed with time, the prognosis of patients seemed to be significantly different. Nadruz et al. [22] conducted a study including 277 patients with HFmrEF. They were divided into two groups according to the changes of LVEF:HF with mid-range ejection fraction and no recovered ejection fraction (HFmEF) and HFrecEF. The results showed that the incidence of composite endpoint events of left ventricular assistant device implantation, heart transplantation, and all-cause mortality in the HFrecEF group was significantly lower than that in the HFmEF group (Hazard ratio [HR]: $0.31 ; 95 \%$ confidence interval [CI]: $0.15-0.67)$. And there was also a trend toward a lower risk of death (HR: 0.48 ; $95 \%$ CI: $0.22-1.05 ; p$ $=0.067$ ) in analyses adjusted for all potential confounders. Lupón et al. [12] followed up the patients for 4.6 years, indicating the all-cause mortality and HF rehospitalization

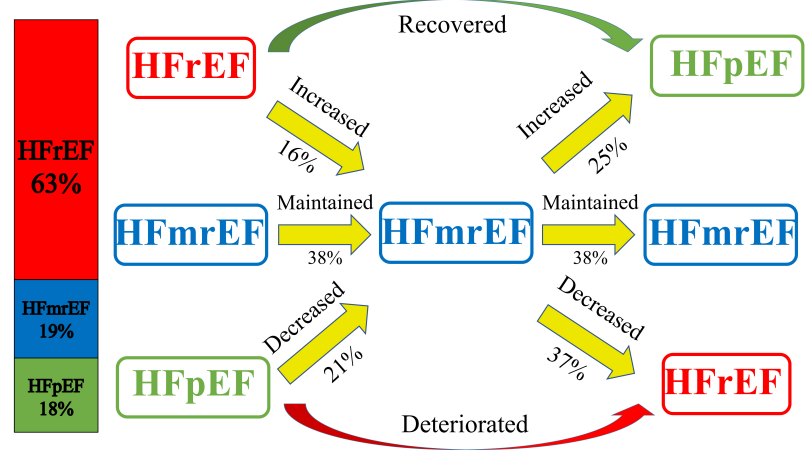

Fig. 2. Illustration of HF types changing with time. The bar chart on the left showed the proportion of the three types of HF. The figure on the right showed the transformation of different types of HF during follow-up [18]. Abbreviations: HF, heart failure; HFrEF, heart failure with decreased ejection fraction; HFm$\mathrm{rEF}$, heart failure with mildly reduced ejection fraction; HFpEF, heart failure with preserved ejection fraction.

rates of HFrEF (HR: 1.74; 95\% CI: 1.31-2.32) and HFpEF (HR: 1.83 ; 95\% CI: 1.27-2.65) were significantly higher than HFrecEF, which demonstrated the prognosis of HFrecEF patients were better. A study conducted by Brann et al. [20] also showed that compared with patients whose LVEF improved from less than $40 \%$ to mid-range levels, patients whose LVEF deteriorated from greater than $50 \%$ 
had a higher risk of all-cause mortality and HF hospitalization (HR: $1.34 ; 95 \% \mathrm{CI}: 1.10-1.82 ; p=0.03$ ) and cardiovascular mortality and HF hospitalization (HR: 1.71; $95 \%$ CI: $1.08-2.50 ; p=0.02)$. Therefore, in patients with HFm$\mathrm{rEF}$, the recovery of LVEF represented a better prognosis, while the deterioration of LVEF represents a worse prognosis, emphasizing the importance of early identification of this part of patients.

\subsubsection{Global longitudinal strain}

Although LVEF was used as the primary inclusion criteria in clinical trials to determine effective drugs and devices for HF patients, this was mainly applicable to HFrEF. And when LVEF was over 45\%, the risk of end-point events was relatively stable with the increase of LVEF [17,23], indicating that LVEF had limitations in assessing prognosis. The strain imaging technique has become a powerful tool for accurately quantifying myocardial structure (including longitudinal, circumferential, radial, and area strain) [24]. The global longitudinal strain (GLS) was considered to be the most reliable parameter, which mainly reflected the contractile function of the subendocardial layer of the left ventricular wall [25] and was sensitive to early disease detection of left ventricular dysfunction [24]. Moreover, GLS was closely related to the prognosis of patients with HF. Chang et al. [26] conducted a retrospective study including $273 \mathrm{HFmrEF}$ patients who were divided into three groups in the follow-up period: HF with worse EF (HFwEF) (LVEF $<40 \%$ ), HF with similar EF (HFsEF) (LVEF: 40-49\%), and HFrecEF (LVEF $>50 \%$ ), indicating that baseline GLS was associated with follow-up LVEF change (HFwEF vs. HFsEF vs. HFrecEF: $-7.8 \%$ vs. $-10.5 \%$ vs. $-17.5 \%, p<0.05)$. This suggests that GLS is of great significance in risk stratification of patients with HFmrEF. A meta-analysis of 16 observational studies showed that the increase in the absolute value of GLS was independently associated with lower mortality (HR: 0.50; 95\% CI: 0.36-0.69) and had a greater impact on mortality than LVEF, emphasizing that monitoring GLS during follow-up was of great significance to comprehensively evaluate the prognosis of patients [27]. In addition, GLS was a better prognostic indicator than LVEF in predicting readmission and malignant arrhythmias in HF. The prognostic value of LVEF was lost in HFmrEF and HFpEF, while GLS had an independent prognostic value in this population. Stanton et al. [28] found that GLS was still an independent risk factor for all-cause death in patients with LVEF $>35 \%$ and can further provide prognostic information based on LVEF. Chang et al. [26] further demonstrated in HFmrEF patients, baseline GLS (HR: 1.26; 95\% CI: $1.17-1.86$ ) and GLS change (HR: 1.12; 95\% CI: $1.03-1.22)$ in the follow-up period were all associated with cardiovascular death. Using $-11 \%$ as a cut-off value in the multivariable analysis, left ventricular GLS $>-11 \%$ was independently associated with cardiovascular death (HR: 3.21; 95\% CI: 1.03-9.95). In conclusion, monitoring GLS plays an important role in evaluating the prognosis of patients with HF, especially in patients with HFmrEF.

\subsection{Cardiac magnetic resonance}

\subsubsection{Late gadolinium enhancement}

Sudden cardiac death (SCD) was one of the leading causes of death in patients with HF, especially in patients with mild symptoms. Implantable cardioverter defibrillator (ICD) implantation was an effective measure for preventing and treating SCD. The 2021 ESC guideline [3] only regarded HFrEF as an indication for ICD implantation and omit HFmrEF and HFpEF. Notably, although patients with LVEF $\leq 35 \%$ were at the highest absolute risk of death, more than $70 \%$ of SCD in patients with CAD occurred in patients with $\mathrm{LVEF}>35 \%$, leaving most subjects at risk largely uncaptured by an LVEF-centered risk stratification [29,30]. This highlights the importance of risk stratification for HFmrEF and HFpEF patients.

Myocardial fibrosis could be caused by a variety of pathological processes, and its existence was related to poor clinical prognosis. CMR can provide a non-invasive assessment of cardiac structure, function, and tissue characteristics, among which late gadolinium enhancement (LGE) was one of the most crucial examination methods for myocardial fibrosis. A prospective study by Halliday et al. [31] including 399 DCM patients with LVEF $\geq 40 \%$ indicated that patients with LGE had a significantly higher risk of SCD than those without LGE (HR: 9.2; 95\% CI: 3.9-21.8). In addition, Klem et al. [32] found in patients with LGE, the incidence of end-point events in patients with LVEF $>30 \%$ was similar to that in patients with $\mathrm{LVEF} \leq 30 \%$, indicating both HFmrEF and HFpEF patients may benefit from ICD implantation. But more studies are still needed to confirm its clinical application.

\subsubsection{T1 mapping}

Although LGE was the gold standard for detecting myocardial fibrosis and evaluating myocardial viability, it can only be analyzed qualitatively and semi-quantitatively. CMR T1 mapping technique was a novel tool that allowed non-invasive quantitative analysis of intercellular and diffuse myocardial fibrosis [33]. The two most common measurements were native T1 and extracellular volume fraction (ECV).

Native T1 reflects the mixed signal of cardiomyocyte and extracellular stroma. Diseases that lead to cardiomyocyte edema (such as acute myocardial infarction, myocarditis) and extracellular interstitial volume increase (such as myocardial fibrosis) can increase native T1. Doeblin et al. [34] demonstrated that compared with healthy controls (972 $\pm 31 \mathrm{~ms})$ and HFpEF (985 $\pm 32 \mathrm{~ms})$, the native T1 of HFmrEF ((1027 $\pm 40 \mathrm{~ms})$ and HFrEF $(1033 \pm$ $54 \mathrm{~ms}$ ) were significantly increased. With respect to prognosis, a study conducted by Puntmann et al. [35] including 637 patients with non-ischemic dilated cardiomyopa- 
thy (NIDCM) showed that native T1 in patients with allcause death was significantly higher than in patients without $(1068 \mathrm{~ms} v s$. $994 \mathrm{~ms}, p<0.001)$. And its ability to predict end-point events was higher than that of LGE. Since the average LVEF of included patients was 43\% (39-46\%), it could be inferred that native T1 may have a strong prognostic value in patients with HFmrEF. However, it should be noted that the normal value of native $\mathrm{T} 1$ varies with the magnetic field strength of the main magnet [36]. In addition, this study only included patients with NIDCM, so further validation in patients with other etiologies was still needed.

Based on the T1 mapping technique, ECV was a new index calculated by obtaining T1 value and hematocrit before and after injection of contrast agent, which used a specific formula to reflect the percentage of extracellular interstitial volume in the whole myocardial volume. Extracellular matrix expansion was a key factor in ventricular remodeling and a potential therapeutic target, while ECV was a marker of myocardial remodeling. A study by Treibel et al. [37] including 1714 patients with HF, with a mean LVEF of 57\% (45-64\%), demonstrated that ECV was independently associated with multiple end-points including allcause death and HF readmission (HR: 1.41, 95\% CI: $1.26-$ 1.59). And there were 547 patients in the LVEF $<50 \%$ subgroup. Further analysis showed that ECV was still an independent risk factor for end-point events (HR: $1.21 ; 95 \% \mathrm{CI}$ : 1.02-1.44), suggesting that ECV may be an important prognostic indicator in HFmrEF. Since this is a relatively new technology, more research is needed.

\subsubsection{Cardiac magnetic resonance feature tracking}

CMR feature tracking (CMR-FT) technique used tissue voxel motion tracking on steady-state free precession images to measure longitudinal, circumferential, and radial left ventricular strain. Similar to STE, CMR-FT was rapid and semi-automated, required no additional scans and sequences, and reduced post-processing time. There was a good correlation between CMR-FT and two dimensional/three dimensional-STE in GLS $(r=0.83$ and 0.87$)$ [38]. Furthermore, CMR-FT could avoid the poor image situation of STE effectively. In different HF groups, GLS measured by CMR-FT was similar to that measured by STE. The absolute value of GLS $(-15.7 \% \pm 2.1 \%)$ in HFm$\mathrm{rEF}$ was significantly lower than that in normal controls and HFpEF patients but higher than that in HFrEF patients [33]. In terms of prognosis, a multicenter study by Romano et al. [39] including 1012 patients of HFmrEF and HFrEF showed that GLS measured by CMR-FT was independently correlated with all-cause death (HR: $1.89 ; p<0.001)$ and could show additional predictive value for all-cause death based on LGE.

\section{Biomarkers}

\section{$4.1 \mathrm{~N}$-Terminal pro-B-type natriuretic peptide}

HF causes cardiac pressure or volume overload, leading to the increased secretion of pro-B-type natriuretic peptide (BNP), which can be split into BNP, with the effect of not only decreasing angiotensin and aldosterone but also causing the vasodilation of arteries, and N-Terminal proBNP (NT-pro BNP) without any biological activity (Fig. 3). NT-pro BNP has been proved to be of diagnostic and prognostic value in patients with HFrEF and HFpEF [40,41]. With respect to HFmrEF, an observational study involving 9847 outpatients with HF [42] showed that the median NT-pro BNP of HFmrEF patients was $1540 \mathrm{pg} / \mathrm{mL}$, lower than $2288 \mathrm{pg} / \mathrm{mL}$ of HFrEF, and higher than $1428 \mathrm{pg} / \mathrm{mL}$ of HFpEF. Although NT-pro BNP in HFmrEF patients was lower than that in HFrEF patients, for prognosis, the risk of all-cause death and HF rehospitalization in HFmrEF patients with higher NT-pro BNP was twice as high as that in patients with lower NT-pro BNP (HR: $2.00 ; 95 \% \mathrm{CI}$ : 1.71-2.34), higher than that in HFrEF (HR: $1.48 ; 95 \%$ CI: 1.36-1.61) and HFpEF (HR: 1.86; 95\% CI: 1.58-2.18). For prognosis, the effect of elevated NT-pro BNP on HFmrEF was higher than that on HFrEF and HFpEF, which may be attributed to the "deterioration" of LVEF in some patients with baseline HFmrEF during follow-up.

\subsection{Cardiac troponin}

The cardiac troponin (cTn) complex consists of three subunits located on the filaments of the striated muscle, namely troponin $\mathrm{T}(\mathrm{TnT})$, troponin $\mathrm{I}(\mathrm{TnI})$, and troponin $\mathrm{C}$ $(\mathrm{TnC}) . \mathrm{TnT}$ is a protein that connects troponin complexes to tropomyosin, while $\mathrm{TnI}$ controls the binding of actin to myosin. Both TnI and TnT have been proved to be specific blood biomarkers of the heart (Fig. 3). The determination of high sensitivity cTn (hs-cTn) can provide a more sensitive measurement value so that a lower concentration can be detected. Moliner et al. [43] found no significant difference in hs-cTnT levels among the three HF types. But for prognosis, compared with HFrEF and HFpEF, the risk of all-cause death and hospitalization for HF in patients with HFmrEF was significantly higher (HR HFrEF $v s$. HFmrEF vs. HFpEF: 1.71 vs. 3.76 vs. 1.87), suggesting that patients with HFmrEF may be more sensitive to mild ischemic injury than patients with HFrEF or HFpEF (Table 2). Moreover, the simultaneous detection of hs-cTnT and NT-pro BNP can further identify high-risk patients [44]. In addition, in patients with normal NT-pro BNP, hs-cTnT was still independently related to the occurrence of adverse events, suggesting hs-cTnT can provide additional prognostic information on the basis of NT-pro BNP [45].

\subsection{Suppression of tumorigenicity 2}

Suppression of tumorigenicity 2 (ST2) is a member of the interleukin (IL)-1 receptor family, mainly in the form of the transmembrane binding receptor (ST2L) and solu- 


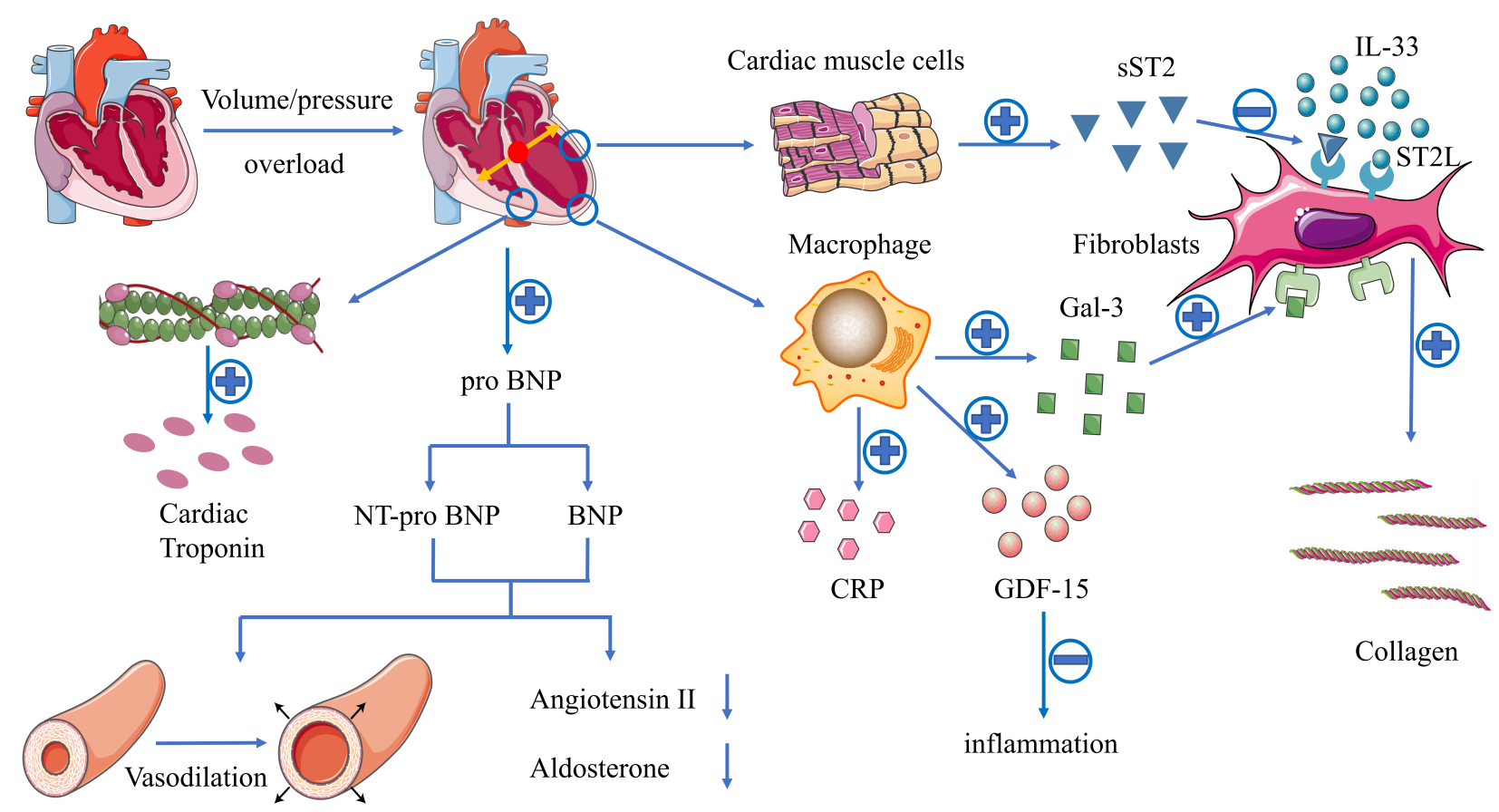

Fig. 3. Illustration of the mechanisms of different biomarkers in HF. HF causes cardiac pressure or volume overload, leading to the increased secretion of pro-BNP, which can be split into BNP, with the effect of not only decreasing angiotensin and aldosterone but also causing the vasodilation of arteries, and NT-pro BNP without any biological activity. ST2 is a member of the IL-1 receptor family, mainly in the form of ST2L and SST2. IL-33 binds o ST2 to produce anti-fibrosis and anti-apoptosis effects. However, under local inflammation and/or mechanical or biochemical stress, the binding of sST2 to ST2L in damaged myocardial tissue blocks the beneficial effects mediated by IL-33, which leads to the decrease of resistance to apoptosis and the increase of myocardial fibrosis. Gal-3 is a soluble $\beta$-galactoside binding glycoprotein, which is released in the myocardium through the paracrine effect. It can further stimulate myofibroblast proliferation and collagen deposition, leading to myocardial fibrosis and cardiac pathological remodeling, while inhibition of Gal-3 expression can reduce cardiac fibrosis and improve left ventricular dysfunction. GDF-15 belongs to the transforming growth factor- $\beta$ superfamily. Under pathological conditions, the expression of GDF-15 can be up-regulated as a response to inflammation, hypoxia, and oxidative stress. CRP has been identified as a classic marker of systemic inflammation, mainly produced by hepatocytes and by cardiovascular tissue in the event of infection, cell invasion, or tissue injury. cTn is the main component of cardiac cardiomyocytes, which would be tested when cardiac injury was experienced. Abbreviations: BNP, B-type natriuretic peptide; NT-pro BNP, N-Terminal pro-B-type natriuretic peptide; sST2, soluble suppression of tumorigenicity 2; IL-33, interleukin-33; Gal-3, galectin-3; GDF-15, growth differentiation factor-15; CRP, C-reactive protein.

ble (sST2). IL-33 binds to ST2L [46] to produce antifibrosis and anti-apoptosis effects [47]. However, under local inflammation and/or mechanical or biochemical stress, the binding of SST2 to ST2L in damaged myocardial tissue blocks the beneficial effect mediated by IL-33, which leads to the decrease of resistance to apoptosis and the increase of myocardial fibrosis (Fig. 3). Therefore, sST2 is a new biomarker reflecting myocardial remodeling and fibrosis which could be used in the case of renal insufficiency [48]. An observational study conducted by Song et al. [49] compared the differences between sST2 among the three types of HF. The results showed that the SST2 levels of the three groups were similar (HFrEF vs. HFmrEF vs. HFpEF: 38.9 vs. 32.7 vs. $40.5 \mathrm{ng} / \mathrm{mL} ; p=0.194)$, which may imply that the degree of myocardial fibrosis may be similar in
HF patients with different LVEF. In patients with elevated baseline sST2 levels, a sustained increase in sST2 levels during follow-up was associated with an increased risk of death [47]. Moliner et al. [43] assessed the relationship between sST2 concentration and prognosis in three HF types. The results demonstrated that $\mathrm{SST} 2$ had a significant prognosis effect in HFrEF and HFmrEF patients. But in HFpEF patients, the effect was not significant (Table 2).

\subsection{Other biomarkers}

Galectin-3 (Gal-3) is a soluble $\beta$-galactoside-binding glycoprotein, which is released in the myocardium through the paracrine effect. It can further stimulate myofibroblast proliferation and collagen deposition, leading to myocardial fibrosis and cardiac pathological remodeling, while 
Table 2. Effect of biomarkers on prognosis of different types of HF.

\begin{tabular}{lccc}
\hline Biomarker & HFrEF & HFmrEF & HFpEF \\
\hline NT-pro BNP & ++ & ++ & ++ \\
sST2 & + & ++ & - \\
Gal-3 & + & ++ & ++ \\
GDF-15 & ++ & $++^{a}$ & ++ \\
hs-cTn & + & ++ & + \\
hs-CRP & + & ++ & - \\
\hline
\end{tabular}

Abbreviations: HFrEF, heart failure with decreased ejection fraction; HFmrEF, heart failure with mildly reduced ejection fraction; HFpEF, heart failure with preserved ejection fraction; NT-pro BNP, N-Terminal proB-type natriuretic peptide; sST2, soluble suppression of tumorigenicity-2; Gal-3, galectin-3; GDF-15, growth differentiation factor-15; hs-cTn, high sensitivity cardiac troponin; hs-CRP, high sensitivity C-reactive protein.

${ }^{a}$ The prognosis effect of GDF-15 has been confirmed in HFpEF and HF with non-reduced EF patients. But until now, there is still no clinical trials focusing only on HFmrEF patients.

inhibition of Gal-3 expression can reduce cardiac fibrosis and improve left ventricular dysfunction [50] (Fig. 3). For clinical application, the 2013 American guideline recommended testing for Gal-3 (IIb class recommendation) in patients with acute and chronic HF for risk stratification and prognosis assessment. A study by Moliner et al. [43] including $1069 \mathrm{HF}$ patients analyzing different types of HF patients demonstrated that there was no significant difference in Gal-3 levels between HFmrEF and HFrEF, while Gal-3 was significantly higher in HFpEF patients. After a follow-up for 4.9 years, the results showed that Gal-3 had a significant predictive value for all-cause death in all three types of HF, and the predictive value increased with the increase of LVEF, suggesting that Gal-3 had a better ability to predict end-point events in patients with HFmrEF (Table 2).

Growth differentiation factor-15 (GDF-15), also known as macrophage inhibitory cytokine-1 (MIC-1), belongs to the transforming growth factor- $\beta$ superfamily. Under physiological conditions, the expression of GDF-15 in human tissues is weak except for the placenta [51], while under pathological conditions, the expression of GDF-15 can be up-regulated by the $p 53$ gene as a response to inflammation, hypoxia, and oxidative stress [52] (Fig. 3). Previous studies have confirmed that GDF-15 had the ability to regulate inflammation, inhibiting apoptosis, and antifibrosis [53]. Chan et al. [54] compared the effects of GDF15 on the prognosis of patients with HFrEF and HFpEF, indicating that even after adjusting NT-pro BNP, GDF-15 was still independently associated with multiple end-points including all-cause death and hospitalization of HF in both groups. However, in the study of Fernandez et al. [55], which compared the role of GDF-15 in HFpEF and HFm$\mathrm{rEF}$ patients, the result indicated that although there was no significant difference between HFpEF and HFmrEF, GDF15 was independently related to all-cause death in HFpEF and HFmrEF. But until now, there are still no clinical trials focusing only on HFmrEF patients, leaving a gap for this part of patients.

C-reactive protein (CRP) has been identified as a classic marker of systemic inflammation, mainly produced by hepatocytes and cardiovascular tissue in the event of infection, cell invasion, or tissue injury [56] (Fig. 3). High sensitivity CRP (hs-CRP) can be detected at a concentration much lower than that of conventional CRP so that it can identify mild inflammation. A post-hoc analysis of PROTECT study [57] including 843 patients with acute HF, showed that CRP there was no significant difference among the three types of HF in patients with chronic HF. For prognosis, Moliner et al. [43] demonstrated that hs-CRP was independently associated with all-cause death and hospitalization of HF in HFmrEF and HFrEF (HR: 1.84 vs. 1.50) and had a higher prognostic significance for patients with HFmrEF compared with HFpEF. However, in patients with HFpEF, CRP cannot predict its prognosis, which was consistent with the results of previous studies [58,59] (Table 2). Obviously, as a marker of systemic inflammation, CRP is likely to be affected by a variety of diseases other than HF, resulting in a decrease in its specificity.

In conclusion, since HFmrEF was proposed, more biomarkers have been used to evaluate HFmrEF. But it should be noticed that only natriuretic peptides and cardiac troponin were mentioned in the latest guideline in 2021 [3], which means that further research is still needed for the other biomarkers above.

\section{Treatment}

At present, there are no clinical trials conducted solely for HFmrEF patients, and the current evidence comes from the subgroup analysis of clinical trials with $\mathrm{HFpEF}$ and HFrEF patients as the study population.

\section{$5.1 \beta$-blockers}

$\beta$-blockers played a role in blocking the direct effect of adrenergic receptors on cardiomyocytes, reducing heart rate, changing vascular function and the neuroendocrine response to HF [60], which were listed as IA recommendation [3] for the treatment of HFrEF. However, whether patients with HFmrEF also benefit from $\beta$-blockers remains unclear. In the OPTIMIZE-HF trial [61], $\beta$-blockers showed no benefit in patients with HFpEF (LVEF $\geq 40 \%$ ). But in SENIORS trial [62], the result demonstrated that nebivolol could significantly reduce all-cause mortality and $\mathrm{CV}$ hospitalization. No interaction was found between LVEF $\leq 35 \%$ and LVEF $>35 \%$ ( $p$ for interaction: 0.42 ). However, in these studies, HFmrEF patients accounted for only a part of all patients. So, in CHART-2 study [13] in- 
cluding 596 HFmrEF patients, the result showed that betablockers improved clinical outcomes and reduced mortality (HR:0.57; 95\% CI: 0.37-0.87). Similar result was found in Swede HF Registry [9], though only HFmrEF patients with CAD were showed significant benefits (HR: 0.74; 95\% CI: 0.59-0.92). A meta-analysis of individual data conducted by Cleland et al. [63] showed the use of $\beta$-blockers could significantly reduce cardiovascular death (HR: 0.48; 95\% CI: 0.24-0.97) in patients with sinus rhythm.

In conclusion, since the HFmrEF firstly proposed in 2016 ESC guideline, more and more studies started to focus on this this subtype of HF. And benefits were found in some conditions such as patients with sinus rhythm or CAD. Since the similar etiologies and comorbidities with HFrEF, the 2021 ESC guideline [3] recommended the use of $\beta$-blockers in HFmrEF patients (Class IIb).

\subsection{Angiotensin converting enzyme inhibitor/Angiotensin receptor blocker}

Since similar characteristics have been found between HFmrEF and HFrEF patients, researchers started to confirm the treatment effort of HFrEF in HFmrEF patients. The CHARM-preserved [64] and I-PRESERVE trial [65] which included patients of LVEF $\geq 40 \%$ and LVEF $\geq 45 \%$, firstly tried to confirm the effort of candesartan and irbesartan. The results showed no significant difference with respect to the primary outcomes of cardiovascular death or HF hospitalization compared with placebo. The same result was found in OPTIMIZED-HF trial [61]. These were possibe because all the trials contained a great number of patients with LVEF $\geq 50 \%$. Since the term HFmrEF was firstly proposed in 2016, studies started to focus on HFmrEF. In the post hoc analysis of PEACE trial included 2512 HFmrEF patients with IHD, the result indicated that trandolapril could significantly reduce all-cause mortality (RR: 0.85; 95\% CI: $0.73-0.99, p=0.03$ ) [66]. In addition, the CHARM study [11] including a total of 1322 HFmrEF patients showed candesartan could significantly reduce the risk of cardiovascular death or hospitalization for HF (HR: 0.76; 95\% CI: 0.61-0.96). The same result was found in the further analysis of SwedeHF Registry [9] which indicated ACEI/ARB could significantly reduce 1-year mortality of HFmrEF patients. Moreover, in patients with HFmrEF, many will also have CAD, HT and will, therefore, already be treated with ACEI/ARB. Therefore, the $2021 \mathrm{ESC}$ guideline [3] recommended the use of ACEI/ARB in HFmrEF patients (Class IIb).

\subsection{Aldosterone receptor antagonist}

The same results were found in another drug recommended by guidelines to treat $\mathrm{HFrEF}$, the mineralocorticoid receptor antagonist (MRA). The TOPCAT trial [67] including patients with $\mathrm{LVEF} \geq 45 \%$ demonstrated that although spironolactone did not significantly improve the prognosis after three years of follow-up, its subgroup analysis showed spironolactone reduced the risk of all-cause death or hospitalization for HF in patients with LVEF range from $45 \%$ to $49 \%$. Furthermore, with the increase of LVEF, the effect of spironolactone on improving prognosis decreased significantly ( $p$ for interaction $=0.046$ ) [68]. In light of these findings from TOPCAT, the 2017 American updated guideline for HF and 2021 ESC guideline added the Class IIb recommendation for HFmrEF patients [3,69].

\subsection{Angiotensin receptor neprilysin inhibitor}

Similarly, the PARAGON-HF trial [70] including patients with LVEF $\geq 45 \%$ also demonstrated sacubitril/valsartan significantly reduced HF hospitalization and cardiovascular mortality (HR: 0.78; 95\% CI: $0.64-0.95$ ) in the LVEF $\leq 57 \%$ subgroup, even it did not improve prognosis in the whole population (Table 3 , Ref. [9,11,13,6165,67,70-74]). The ongoing PARALLAX trial [75] further included patients with $\mathrm{LVEF} \geq 40 \%$, with a reduction of NT-pro BNP as the primary end-point event, expecting to have promising results in HFmrEF patients. Based on this result, it has been approved by Food and Drug Administration as an extended indication in those with LVEF 'less than normal', which including HFmrEF patients. Given the above evidence, ANRI was recommended IIb class for HFmrEF patients [3].

\subsection{Sodium-glucose cotransporter 2 inhibitor}

Sodium-glucose cotransporter 2 inhibitor (SGLT2i) was a newly emerging drug used for improving glycaemic control in patients with DM by decreasing renal glucose reabsorption and thereby increasing urinary glucose excretion, which was found to be effective in HF patients recently [76]. The EMPEROR-Reduced trial [71], which consists of patients with LVEF less than $40 \%$, demonstrated that empagliflozin reduced the primary composite outcomes of death from cardiovascular causes or hospitalization for HF (HR: 0.75; 95\% CI: 0.65-0.86; $p<$ $0.001)$ whether or not diabetes was combined, which was consistent with results of the DAPA-HF trial [72] of another SGLT2i, dapagliflozin. In the subgroup analysis of EMPEROR-Reduced trial [76] of patients with LVEF ranged from $30 \%$ to $40 \%$, there was no significant benefit (HR: 0.99; 95\% CI: 0.76-1.31) compared with placebo, which contrasted to the results of DAPA-HF trial (HR: 0.81; 95\% CI: 0.65-0.99) [72].

The SOLOIST-WHF trial conducted in 2020 including HFrEF, HFmrEF, and HFpEF patients, showed that among patients with DM who had worsening HF, the end-point events of the total number of cardiovascular deaths, hospitalizations and urgent visits for HF were significantly lower with sotagliflozin than with placebo [73]. The results were still consistent in patients with both HFpEF (LVEF $\geq 50 \%$ ) and combined HFmrEF and HFrEF (LVEF < 50\%) patients. Although such patients were enrolled in the trial, there was no evidence of heterogeneity of treatment effect according 
Table 3. Clinical evidence of different drugs in HFmrEF patients.

\begin{tabular}{|c|c|c|c|c|c|c|c|c|}
\hline Study & Type & Drugs & Control & LVEF & $\mathrm{HR} / \mathrm{RR}$ & $95 \% \mathrm{CI}$ & Outcome & $p$ value \\
\hline \multicolumn{9}{|l|}{ ACEI/ARB } \\
\hline CHARM-preserved [64] & $\mathrm{RCT}$ & Candesartan & placebo & $>40 \%$ & 0.89 & $0.77-1.03$ & cardiovascular death + hospitalization for $\mathrm{HF}$ & 0.118 \\
\hline I-PRESERVE [65] & $\mathrm{RCT}$ & Irbesartan & placebo & $45-59 \%$ & 0.98 & $0.85-1.12$ & all-cause mortality + hospitalization for cardiovascular cause & 0.28 \\
\hline CHARM [11] & $\mathrm{RCT}$ & Candesartan & placebo & $40-49 \%$ & 0.76 & $0.61-0.96$ & cardiovascular death + hospitalization for $\mathrm{HF}+$ & 0.02 \\
\hline PEACE (post hoc analysis) [66] & $\mathrm{RCT}$ & Trandolapril & placebo & $40-50 \%$ & 0.79 & $0.63-0.98$ & all-cause mortality + nonfatal MI + stroke & 0.03 \\
\hline Swede HF [9] & Registry & ACEI/ARB & - & $40-49 \%$ & $\begin{array}{l}0.56 \\
0.63\end{array}$ & $\begin{array}{l}0.50-0.62 \\
0.54-0.75\end{array}$ & all-cause mortality & $<0.001$ \\
\hline \multicolumn{9}{|l|}{$\beta$-blocker } \\
\hline OPTIMIZED-HF [61] & Registry & $\beta$-blocker & no $\beta$-blocker & $\geq 40 \%$ & 1.21 & $0.87-1.68$ & mortality at $60-90$ days & 0.255 \\
\hline CHART-2 [13] & Registry & $\beta$-blocker & no $\beta$-blocker & $40-49 \%$ & 0.57 & $0.37-0.87$ & mortality & 0.010 \\
\hline SENIORS [62] & RCT & Nebivolol & placebo & all & 0.86 & $0.74-0.99$ & all-cause mortality + cardiovascular hospitalization & 0.42 \\
\hline Swede HF [9] & Registry & $\beta$-blocker & no $\beta$-blocker & $40-49 \%$ & $\begin{array}{l}0.74 \\
0.99\end{array}$ & $\begin{array}{l}0.59-0.92 \\
0.78-1.26\end{array}$ & all-cause mortality & $\begin{array}{l}0.006 \\
0.944\end{array}$ \\
\hline meta-analysis [63] & & $\beta$-blocker & placebo & $40-49 \%$ & 0.48 & $0.24-0.97$ & cardiovascular death & 0.04 \\
\hline \multicolumn{9}{|l|}{ MRA } \\
\hline TOPCAT [67] & RCT & Spironolactone & placebo & $45-50 \%$ & 0.72 & $0.50-1.05$ & all-cause death + hospitalization for heart failure & 0.046 \\
\hline \multicolumn{9}{|c|}{ ( } \\
\hline PARAGON-HF [70] & RCT & Sacubitril/valsartan & valsartan & $45-57 \%$ & 0.78 & $0.64-0.95$ & cardiovascular death + hospitalization for heart failure & $<0.05$ \\
\hline \multicolumn{9}{|l|}{ SGLT2 inhibitor } \\
\hline EMPEROR-Reduced [71] & $\mathrm{RCT}$ & Empagliflozin & placebo & $\leq 40 \%$ & 0.75 & $0.65-0.86$ & cardiovascular death + hospitalization for $\mathrm{HF}$ & $<0.05$ \\
\hline DAPA-HF [72] & $\mathrm{RCT}$ & Dapagliflozin & placebo & $\leq 40 \%$ & 0.74 & $0.65-0.85$ & worsening HF + cardiovascular death & $<0.05$ \\
\hline SOLOIST-WHF [73] & $\mathrm{RCT}$ & Sotagliflozin & placebo & $\begin{array}{l}\geq 50 \% \\
<50 \%\end{array}$ & $\begin{array}{l}0.48 \\
0.72\end{array}$ & $\begin{array}{l}0.27-0.86 \\
0.56-0.94\end{array}$ & $\begin{array}{l}\text { total number of deaths from cardiovascular causes }+ \\
\text { hospitalizations and urgent visits for heart failure }\end{array}$ & - \\
\hline EMPEROR-Preserved [74] & RCT & Empagliflozin & placebo & $\begin{array}{l}40-50 \% \\
50-60 \%\end{array}$ & $\begin{array}{l}0.71 \\
0.80\end{array}$ & $\begin{array}{l}(0.57-0.88) \\
(0.64-0.99)\end{array}$ & cardiovascular death + hospitalization for $\mathrm{HF}$ & $<0.05$ \\
\hline
\end{tabular}

Abbreviations: HFmrEF, heart failure with mildly reduced ejection fraction; MI, myocardium infarction; HF, heart failure; LVEF, left ventricular ejection fraction; HR, hazard ratio; RR, risk ratio; $\mathrm{CI}$, confidence interval. 
to LVEF, early termination of the trial and the small sample size of this subgroup made it difficult to draw any firm conclusion in this regard. To further clarify the efficacy of SGLT $2 \mathrm{i}$ in patients with LVEF $>40 \%$, the EMPERORPreserved trial [74] was conducted, firstly indicating empagliflozin could significantly reduce composite end-point events of hospitalization for HF or cardiovascular death not only in patients with LVEF between $40 \%$ and $50 \%$, but also in patients with LVEF in the range of 50-60\% (Table 3). The results were inspiring for patients of HFmrEF and HF$\mathrm{pEF}$, and also brought hope for the application of other SGLT2i in these patients. The ongoing studies including PRESERVED-HF, and DELIVER will further assess the effects of SGLT2 inhibitors in HFmrEF and HFpEF.

\section{Conclusions}

Since the introduction of HFmrEF as an HF category in 2016 ESC guideline, a considerable number of clinical studies have included patients in the "grey area", giving us a preliminary understanding of this field. HFmrEF represents not only the intermediate category between HFrEF and HFpEF but also the patient group with different clinical characteristics and prognosis. HFmrEF resembles a transitional stage between HFrEF and HFpEF, including HFwEF, HF$\mathrm{sEF}$, and HFrecEF, which have different prognosis. Therefore, it is of great significance to find appropriate indicators to identify each type of HFmrEF.

In today's era of precision medicine, new advances in HFmrEF treatment may be further extended to identify the characteristics of each HF patient, and these characteristics may help to further improve risk stratification. At present, cardiovascular imaging has developed from obtaining qualitative diagnostic information to more quantitative evaluation methods. Advanced imaging methods, including 2DSTE, 3D-STE, T1 mapping, and CMR-FT, et al., have been proved to have the potential to identify high-risk patients in HFmrEF, and the emergence of new biomarkers have been proved to provide additional predictive value. Multiple time points and combined detection of various indicators will also be the development direction of precision therapy in the future.

\section{Author contributions}

Conceptualization-WG; investigation-ZS; writing - original draft preparation-ZS; writing - review and editing - XW and WG; project administration - XW; funding acquisition-WG; All authors have read and agreed to the published version of the manuscript.

\section{Ethics approval and consent to participate}

Not applicable.

\section{Acknowledgment}

We would like to express our gratitude to all those who helped us during the writing of this manuscript. Thanks to all the peer reviewers for their opinions and suggestions.

\section{Funding}

This work was supported by grants from the Peking University Health Science Center - University of Michigan Joint Institute for Translational and Clinical Research (grant No. BMU2020JI009), and the National Natural Science Foundation of China (grant No. 81972149, 81871850), Beijing Natural Science Foundation (grant No. 7212125).

\section{Conflict of interest}

The authors declare no conflict of interest.

\section{References}

[1] Ponikowski P, Anker SD, AlHabib KF, Cowie MR, Force TL, Hu $\mathrm{S}$, et al. Heart failure: preventing disease and death worldwide. ESC Heart Failure. 2014; 1: 4-25.

[2] Ponikowski P, Voors AA, Anker SD, Bueno H, Cleland JGF, Coats AJS, et al. 2016 ESC Guidelines for the diagnosis and treatment of acute and chronic heart failure: The Task Force for the diagnosis and treatment of acute and chronic heart failure of the European Society of Cardiology (ESC) Developed with the special contribution of the Heart Failure Association (HFA) of the ESC. European Heart Journal. 2016; 37: 2129-2200.

[3] McDonagh TA, Metra M, Adamo M, Gardner RS, Baumbach A, Böhm M, et al. 2021 ESC Guidelines for the diagnosis and treatment of acute and chronic heart failure. European Heart Journal. 2021; 42: 3599-3726.

[4] Ge Z, Li A, McNamara J, dos Remedios C, Lal S. Pathogenesis and pathophysiology of heart failure with reduced ejection fraction: translation to human studies. Heart Failure Reviews. 2019; 24: 743-758.

[5] Lakhani I, Leung KSK, Tse G, Lee APW. Novel mechanisms in heart failure with preserved, midrange, and reduced ejection fraction. Frontiers in Physiology. 2019; 10: 874.

[6] Kapoor JR, Kapoor R, Ju C, Heidenreich PA, Eapen ZJ, Hernandez AF, et al. Precipitating Clinical Factors, Heart Failure Characterization, and Outcomes in Patients Hospitalized with Heart Failure with Reduced, Borderline, and Preserved Ejection Fraction. JACC: Heart Failure. 2016; 4: 464-472.

[7] Rickenbacher P, Kaufmann BA, Maeder MT, Bernheim A, Goetschalckx K, Pfister O, et al. Heart failure with mid-range ejection fraction: a distinct clinical entity? Insights from the Trial of Intensified versus standard Medical therapy in Elderly patients with Congestive Heart Failure (TIME-CHF). European Journal of Heart Failure. 2017; 19: 1586-1596.

[8] Chioncel O, Lainscak M, Seferovic PM, Anker SD, CrespoLeiro MG, Harjola V, et al. Epidemiology and one-year outcomes in patients with chronic heart failure and preserved, midrange and reduced ejection fraction: an analysis of the ESC Heart Failure Long-Term Registry. European Journal of Heart Failure. 2017; 19: 1574-1585.

[9] Koh AS, Tay WT, Teng THK, Vedin O, Benson L, Dahlstrom $\mathrm{U}$, et al. A comprehensive population-based characterization of heart failure with mid-range ejection fraction. European Journal of Heart Failure. 2017; 19: 1624-1634.

[10] Löfman I, Szummer K, Dahlström U, Jernberg T, Lund LH. Associations with and prognostic impact of chronic kidney disease in heart failure with preserved, mid-range, and reduced ejection 
fraction. European Journal of Heart Failure. 2017; 19: 16061614.

[11] Lund LH, Claggett B, Liu J, Lam CS, Jhund PS, Rosano GM, et al. Heart failure with mid-range ejection fraction in CHARM: characteristics, outcomes and effect of candesartan across the entire ejection fraction spectrum. European Journal of Heart Failure. 2018; 20: 1230-1239.

[12] Lupón J, Díez-López C, de Antonio M, Domingo M, Zamora E, Moliner P, et al. Recovered heart failure with reduced ejection fraction and outcomes: a prospective study. European Journal of Heart Failure. 2017; 19: 1615-1623.

[13] Tsuji K, Sakata Y, Nochioka K, Miura M, Yamauchi T, Onose $\mathrm{T}$, et al. Characterization of heart failure patients with mid-range left ventricular ejection fraction-a report from the CHART-2 Study. European Journal of Heart Failure. 2017; 19: 1258-1269.

[14] Trevisan L, Cautela J, Resseguier N, Laine M, Arques S, Pinto $\mathrm{J}$, et al. Prevalence and characteristics of coronary artery disease in heart failure with preserved and mid-range ejection fractions: a systematic angiography approach. Archives of Cardiovascular Diseases. 2018; 111: 109-118.

[15] Johansson I, Dahlström U, Edner M, Näsman P, Rydén L, Norhammar A. Type 2 diabetes and heart failure: Characteristics and prognosis in preserved, mid-range and reduced ventricular function. Diabetes and Vascular Disease Research. 2018; 15: 494-503.

[16] Sartipy U, Dahlström U, Fu M, Lund LH. Atrial Fibrillation in Heart Failure with Preserved, Mid-Range, and Reduced Ejection Fraction. JACC: Heart Failure. 2017; 5: 565-574.

[17] Solomon SD, Anavekar N, Skali H, McMurray JJV, Swedberg $\mathrm{K}$, Yusuf S, et al. Influence of Ejection Fraction on Cardiovascular Outcomes in a Broad Spectrum of Heart Failure Patients. Circulation. 2005; 112: 3738-3744.

[18] Savarese G, Vedin O, D'Amario D, Uijl A, Dahlström U, Rosano $\mathrm{G}$, et al. Prevalence and Prognostic Implications of Longitudinal Ejection Fraction Change in Heart Failure. JACC: Heart Failure. 2019; 7: 306-317.

[19] Branca L, Sbolli M, Metra M, Fudim M. Heart failure with mid $\square$ range ejection fraction: pro and cons of the new classification of Heart Failure by European Society of Cardiology guidelines. ESC Heart Failure. 2020; 7: 381-399.

[20] Brann A, Janvanishstaporn S, Greenberg B. Association of Prior Left Ventricular Ejection Fraction with Clinical Outcomes in Patients with Heart Failure with Midrange Ejection Fraction. JAMA Cardiology. 2020; 5: 1027-1035.

[21] Rastogi A, Novak E, Platts AE, Mann DL. Epidemiology, pathophysiology and clinical outcomes for heart failure patients with a mid-range ejection fraction. European Journal of Heart Failure. 2017; 19: 1597-1605.

[22] Nadruz W, West E, Santos M, Skali H, Groarke JD, Forman DE, et al. Heart Failure and Midrange Ejection Fraction. Circulation: Heart Failure. 2016; 9: e002826.

[23] Curtis JP, Sokol SI, Wang Y, Rathore SS, Ko DT, Jadbabaie F, et al. The association of left ventricular ejection fraction, mortality, and cause of death in stable outpatients with heart failure. Journal of the American College of Cardiology. 2003; 42: 736742.

[24] Nesbitt GC, Mankad S, Oh JK. Strain imaging in echocardiography: methods and clinical applications. The International Journal of Cardiovascular Imaging. 2009; 25: 9-22.

[25] Lumens J, Prinzen FW, Delhaas T. Longitudinal Strain: 'Think globally, track locally". JACC: Cardiovascular Imaging. 2015; 8: 1360-1363.

[26] Chang W, Lin CH, Hong C, Liao C, Liu Y, Chen Z, et al. The predictive value of global longitudinal strain in patients with heart failure mid-range ejection fraction. Journal of Cardiology. 2021; 77: 509-516.
[27] Kalam K, Otahal P, Marwick TH. Prognostic implications of global LV dysfunction: a systematic review and meta-analysis of global longitudinal strain and ejection fraction. Heart. 2014; 100: $1673-1680$.

[28] Stanton T, Leano R, Marwick TH. Prediction of all-Cause Mortality from Global Longitudinal Speckle Strain. Circulation: Cardiovascular Imaging. 2009; 2: 356-364.

[29] Stecker EC, Vickers C, Waltz J, Socoteanu C, John BT, Mariani $\mathrm{R}$, et al. Population-Based Analysis of Sudden Cardiac Death with and without Left Ventricular Systolic Dysfunction: twoyear findings from the Oregon Sudden Unexpected Death Study. Journal of the American College of Cardiology. 2006; 47: 11611166.

[30] Chatterjee NA, Moorthy MV, Pester J, Schaecter A, Panicker GK, Narula D, et al. Sudden Death in Patients with Coronary Heart Disease without Severe Systolic Dysfunction. JAMA Cardiology. 2018; 3: 591.

[31] Halliday BP, Gulati A, Ali A, Guha K, Newsome S, Arzanauskaite $\mathrm{M}$, et al. Association between midwall late gadolinium enhancement and sudden cardiac death in patients with dilated cardiomyopathy and mild and moderate left ventricular systolic dysfunction. Circulation. 2017; 135: 2106-2115.

[32] Klem I, Weinsaft JW, Bahnson TD, Hegland D, Kim HW, Hayes B, et al. Assessment of Myocardial Scarring Improves Risk Stratification in Patients Evaluated for Cardiac Defibrillator Implantation. Journal of the American College of Cardiology. 2012; 60: 408-420.

[33] Rommel K, Lücke C, Lurz P. Diagnostic and Prognostic Value of CMR T 1 -Mapping in Patients with Heart Failure and Preserved Ejection Fraction. Revista EspañOla De Cardiología. 2017; 70: 848-855.

[34] . Doeblin P, Hashemi D, Tanacli R, Lapinskas T, Gebker R, Stehning C, et al. CMR tissue characterization in patients with HFmrEF. Journal of Clinical Medicine. 2019; 8:1877.

[35] Puntmann VO, Carr-White G, Jabbour A, Yu C, Gebker R, Kelle $\mathrm{S}$, et al. T1-Mapping and Outcome in Nonischemic Cardiomyopathy: all-cause mortality and heart failure. JACC: Cardiovascular Imaging. 2016; 9: 40-50.

[36] Dabir D, Child N, Kalra A, Rogers T, Gebker R, Jabbour A, et $a l$. Reference values for healthy human myocardium using a $\mathrm{T} 1$ mapping methodology: results from the International T1 Multicenter cardiovascular magnetic resonance study. Journal of Cardiovascular Magnetic Resonance. 2014; 16: 69.

[37] Treibel TA, Fridman Y, Bering P, Sayeed A, Maanja M, Frojdh $\mathrm{F}$, et al. Extracellular Volume Associates with Outcomes more Strongly than Native or Post-Contrast Myocardial T1. JACC: Cardiovascular Imaging. 2020; 13: 44-54.

[38] Obokata M, Nagata Y, Wu VC, Kado Y, Kurabayashi M, Otsuji $\mathrm{Y}$, et al. Direct comparison of cardiac magnetic resonance feature tracking and 2D/3D echocardiography speckle tracking for evaluation of global left ventricular strain. European Heart Journal - Cardiovascular Imaging. 2016; 17: 525-532.

[39] Romano S, Judd RM, Kim RJ, Kim HW, Klem I, Heitner JF, et $a l$. Feature-Tracking Global Longitudinal Strain Predicts Death in a Multicenter Population of Patients with Ischemic and Nonischemic Dilated Cardiomyopathy Incremental to Ejection Fraction and Late Gadolinium Enhancement. JACC: Cardiovascular Imaging. 2018; 11: 1419-1429.

[40] Savarese G, Musella F, D'Amore C, Vassallo E, Losco T, Gambardella F, et al. Changes of Natriuretic Peptides Predict Hospital Admissions in Patients with Chronic Heart Failure: a metaanalysis. JACC: Heart Failure. 2014; 2: 148-158.

[41] Cleland JGF, Taylor J, Freemantle N, Goode KM, Rigby AS, Tendera M. Relationship between plasma concentrations of Nterminal pro brain natriuretic peptide and the characteristics and outcome of patients with a clinical diagnosis of diastolic heart 
failure: a report from the PEP-CHF study. European Journal of Heart Failure. 2012; 14: 487-494.

[42] Savarese G, Orsini N, Hage C, Dahlström U, Vedin O, Rosano GMC, et al. Associations with and Prognostic and Discriminatory Role of N-Terminal Pro-B-Type Natriuretic Peptide in Heart Failure with Preserved Versus Mid-range Versus Reduced Ejection Fraction. Journal of Cardiac Failure. 2018; 24: 365374.

[43] Moliner P, Lupón J, Barallat J, de Antonio M, Domingo M, Núñez J, et al. Bio-profiling and bio-prognostication of chronic heart failure with mid-range ejection fraction. International Journal of Cardiology. 2018; 257: 188-192.

[44] Kociol RD, Pang PS, Gheorghiade M, Fonarow GC, O'Connor $\mathrm{CM}$, Felker GM. Troponin elevation in heart failure prevalence, mechanisms, and clinical implications. Journal of the American College of Cardiology. 2010; 56: 1071-1078.

[45] Latini R, Masson S, Anand IS, Missov E, Carlson M, Vago T, et al. Prognostic Value of very Low Plasma Concentrations of Troponin T in Patients with Stable Chronic Heart Failure. Circulation. 2007; 116: 1242-1249.

[46] Miller AM, Liew FY. The IL-33/ST2 pathway - a new therapeutic target in cardiovascular disease. Pharmacology \& Therapeutics. 2011; 131: 179-186.

[47] Seki K, Sanada S, Kudinova AY, Steinhauser ML, Handa V, Gannon; J, et al. Interleukin-33 Prevents Apoptosis and Improves Survival after Experimental Myocardial Infarction through ST2 Signaling. Circulation: Heart Failure. 2009; 2: 684-691.

[48] Bayes-Genis A, Zamora E, de Antonio M, Galán A, Vila J, Urrutia A, et al. Soluble ST2 Serum Concentration and Renal Function in Heart Failure. Journal of Cardiac Failure. 2013; 19: 768 775.

[49] Song Y, Li F, Xu Y, Liu Y, Wang Y, Han X, et al. Prognostic value of sST2 in patients with heart failure with reduced, midrange and preserved ejection fraction. International Journal of Cardiology. 2020; 304: 95-100.

[50] Yu L, Ruifrok WPT, Meissner M, Bos EM, van Goor H, Sanjabi $\mathrm{B}$, et al. Genetic and Pharmacological Inhibition of Galectin3 Prevents Cardiac Remodeling by Interfering with Myocardial Fibrogenesis. Circulation: Heart Failure. 2013; 6: 107-117.

[51] Corre J, Hébraud B, Bourin P. Concise Review: Growth Differentiation Factor 15 in Pathology: a Clinical Role? Stem Cells Translational Medicine. 2013; 2: 946-952.

[52] Vousden KH, Lane DP. P53 in health and disease. Nature Reviews Molecular Cell Biology. 2007; 8: 275-283.

[53] Mimeault M, Batra SK. Divergent molecular mechanisms underlying the pleiotropic functions of macrophage inhibitory cytokine-1 in cancer. Journal of Cellular Physiology. 2010; 224: 626-635.

[54] Chan MMY, Santhanakrishnan R, Chong JPC, Chen Z, Tai BC, Liew OW, et al. Growth differentiation factor 15 in heart failure with preserved vs. reduced ejection fraction. European Journal of Heart Failure. 2016; 18: 81-88.

[55] Mendez Fernandez AB, Ferrero $\square$ Gregori A, Garcia $\square$ Osuna A, Mirabet $\square$ Perez S, Pirla $\square$ Buxo MJ, Cinca $\square$ Cuscullola J, et al. Growth differentiation factor 15 as mortality predictor in heart failure patients with non $\square$ reduced ejection fraction. ESC Heart Failure. 2020; 7: 2223-2229.

[56] Libby P. Inflammation in atherosclerosis. Nature. 2002; 420 : 868-874.

[57] Tromp J, Khan MAF, Mentz RJ, O’Connor CM, Metra M, Dittrich HC, et al. Biomarker Profiles of Acute Heart Failure Patients with a Mid-Range Ejection Fraction. JACC: Heart Failure. 2017; 5: 507-517.

[58] Michowitz Y, Arbel Y, Wexler D, Sheps D, Rogowski O, Shapira $\mathrm{I}$, et al. Predictive value of high sensitivity CRP in patients with diastolic heart failure. International Journal of Cardiology. 2008; 125: 347-351.

[59] Tromp J, Khan MAF, Klip IT, Meyer S, de Boer RA, Jaarsma T, et al. Biomarker Profiles in Heart Failure Patients with Preserved and Reduced Ejection Fraction. Journal of the American Heart Association. 2017; 6: e003989.

[60] von Lueder TG, Kotecha D, Atar D, Hopper I. Neurohormonal blockade in heart failure. Cardiac Failure Review. 2017; 3: 1924.

[61] Fonarow GC, Stough WG, Abraham WT, Albert NM, Gheorghiade M, Greenberg BH, et al. Characteristics, Treatments, and Outcomes of Patients with Preserved Systolic Function Hospitalized for Heart Failure: a report from the OPTIMIZE-HF Registry. Journal of the American College of Cardiology. 2007; 50: 768-777.

[62] Flather MD, Shibata MC, Coats AJS, Van Veldhuisen DJ, Parkhomenko A, Borbola J, et al. Randomized trial to determine the effect of nebivolol on mortality and cardiovascular hospital admission in elderly patients with heart failure (SENIORS). European Heart Journal. 2005; 26: 215-225.

[63] Cleland JGF, Bunting KV, Flather MD, Altman DG, Holmes $\mathrm{J}$, Coats AJS, et al. Beta-blockers for heart failure with reduced, mid-range, and preserved ejection fraction: an individual patient-level analysis of double-blind randomized trials. European Heart Journal. 2018; 39: 26-35.

[64] Yusuf S, Pfeffer MA, Swedberg K, Granger CB, Held P, McMurray JJ, et al. Effects of candesartan in patients with chronic heart failure and preserved left-ventricular ejection fraction: the CHARM-Preserved Trial. The Lancet. 2003; 362: 777-781.

[65] Massie BM, Carson PE, McMurray JJ, Komajda M, McKelvie R, Zile MR, et al. Irbesartan in Patients with Heart Failure and Preserved Ejection Fraction. New England Journal of Medicine. 2008; 359: 2456-2467.

[66] Alzahrani T, Tiu J, Panjrath G, Solomon A. The effect of angiotensin-converting enzyme inhibitors on clinical outcomes in patients with ischemic cardiomyopathy and midrange ejection fraction: a post hoc subgroup analysis from the PEACE trial. Therapeutic Advances in Cardiovascular Disease. 2018; 12: $351-359$.

[67] Pitt B, Pfeffer MA, Assmann SF, Boineau R, Anand IS, Claggett $\mathrm{B}$, et al. Spironolactone for Heart Failure with Preserved Ejection Fraction. New England Journal of Medicine. 2014; 370: 1383-1392.

[68] Solomon SD, Claggett B, Lewis EF, Desai A, Anand I, Sweitzer $\mathrm{NK}$, et al. Influence of ejection fraction on outcomes and efficacy of spironolactone in patients with heart failure with preserved ejection fraction. European Heart Journal. 2016; 37: 455-462.

[69] Yancy CW, Jessup M, Bozkurt B, Butler J, Casey DE Jr, Colvin MM, et al. 2017 ACC/AHA/HFSA focused update of the 2013 $\mathrm{ACCF} / \mathrm{AHA}$ guideline for the management of heart failure: a report of the American College of Cardiology/American Heart Association task force on clinical practice guidelines and the Heart Failure Society of America. Journal of the American College of Cardiology. 2017; 70: 776-803.

[70] Solomon SD, McMurray JJV, Anand IS, Ge J, Lam CSP, Maggioni AP, et al. Angiotensin-neprilysin inhibition in heart failure with preserved ejection fraction. The New England Journal of Medicine. 2019; 381: 1609-1620.

[71] Packer M, Anker SD, Butler J, Filippatos G, Pocock SJ, Carson $\mathrm{P}$, et al. Cardiovascular and renal outcomes with empagliflozin in heart failure. The New England Journal of Medicine. 2020; 383: 1413-1424.

[72] McMurray JJV, Solomon SD, Inzucchi SE, Kober L, Kosiborod $\mathrm{MN}$, Martinez FA, et al. Dapagliflozin in patients with heart failure and reduced ejection fraction. The New England Journal of 
Medicine. 2019; 381: 1995-2008.

[73] Bhatt DL, Szarek M, Steg PG, Cannon CP, Leiter LA, McGuire DK, et al. Sotagliflozin in Patients with Diabetes and Recent Worsening Heart Failure. New England Journal of Medicine. 2021; 384: 117-128.

[74] Anker SD, Butler J, Filippatos G, Ferreira JP, Bocchi E, Böhm $\mathrm{M}$, et al. Empagliflozin in Heart Failure with a Preserved Ejection Fraction. New England Journal of Medicine. 2021; 385: $1451-1461$.
[75] Wachter R, Shah SJ, Cowie MR, Szecsödy P, Shi V, Ibram G, et $a l$. Angiotensin receptor neprilysin inhibition versus individualized RAAS blockade: design and rationale of the PARALLAX trial. ESC Heart Failure. 2020; 7: 856-864.

[76] Grempler R, Thomas L, Eckhardt M, Himmelsbach F, Sauer A, Sharp DE, et al. Empagliflozin, a novel selective sodium glucose cotransporter-2 (SGLT-2) inhibitor: characterisation and comparison with other SGLT-2 inhibitors. Diabetes, Obesity and Metabolism. 2012; 14: 83-90. 


\section{Temporary page!}

${ }_{\mathrm{HT}} \mathrm{T}$ was unable to guess the total number of pages correctly. As there was some unprocessed data that should have been added to the final page this extra page has been added to receive it.

If you rerun the document (without altering it) this surplus page will go away, because ${ }_{\mathrm{AT}} \mathrm{T} \mathrm{X}$ now knows how many pages to expect for this document. 